\title{
Nucleon and deuteron induced spallation reactions
}

\author{
J. Cugnon ${ }^{\mathrm{a}}$, C. Volant ${ }^{\mathrm{b}}, \mathrm{S}$. Vuillier ${ }^{\mathrm{b}}$ \\ a University of Liège, Physics Department, B5, B-4000 Sart-Tilman Liège 1, Belgium \\ ${ }^{\circ}$ CEA DAPNIA/SPhN, CE Saclay, F-91191 Gif-sur-Yvette Cedex, France
}

Received 27 June 1997; revised 18 August 1997; accepted 29 September 1997

\begin{abstract}
The intranuclear cascade (INC) +evaporation model is used to display the gross features of proton, neutron and deuteron induced spallation reactions between $200 \mathrm{MeV}$ and $2 \mathrm{GeV}$ incident energy per nucleon. Particular attention is focused on average particle multiplicities and their dependencies upon target mass number and incident energy. Simple formulae are derived to allow an easy use of these results. They are expected to provide a rapid survey of the gross features of spallation reactions. The decomposition of particle multiplicities and of cross-sections into cascade and evaporation components is performed and discussed. The impact parameter dependence is investigated. The excitation energy left in the target at the end of the cascade step is also studied, as well as the gross properties of the subsequent evolution of the target remnant. It is shown that neutron differential cross-sections can be split into components with reasonably identified physical origin, which exhibit simple power laws as functions of the target mass number. (C) 1997 Elsevier Science B.V.
\end{abstract}

PACS: $25.40 . \mathrm{Sc} ; 25.50 .-\mathrm{h} ; 28.20 .-\mathrm{v}$

Keywords: Nucleon and deuteron-induced reactions; Intranuclear cascade; Evaporation; Particle yields; Target remnant

\section{Introduction}

Spallation reactions are usually defined as the processes by which an incident hadron of relatively high energy interacts with a nuclear target and expels from the latter several particles, mainly nucleons, but also, with a lesser yield, composites and pions. These phenomena are known for a long time. They have been discovered some fifty years ago at the $180 \mathrm{MeV}$ cyclotron at Berkeley [1]. Soon after, a theoretical picture was proposed by Serber [2]. He suggested that the incident particle collides successively and distinctively with several nucleons, losing so a large fraction of its energy. The target becomes well excited and emits a few nucleons. This rough, but basically right, 
picture was substantiated by the so-called intranuclear cascade (INC) plus evaporation model. The predictions of the latter are rather successful for proton-induced reactions in the $\mathrm{GeV}$ range, extending from $\sim 200 \mathrm{MeV}$ to $\sim 2 \mathrm{GeV}$ [3-5]. In this range, a more precise picture has emerged [6]. The incident particle makes a few hard two body collisions. Some of the struck nucleons are ejected with relatively large kinetic energy in a rather short time scale. The other ones make secondary collisions in their way out. Progressively, the (average) number of ejected particles and their mean energy decrease. After some time, the characteristic values of these quantities are similar to those of evaporation, indicating the beginning of this process.

Recently, the interest in the study of spallation reactions has been revived, primarily, but not only, by the advent of new projects in nuclear technology. Among these, let us mention the conception of so-called spallation sources [7] and of accelerator-driven nuclear reactors for energy production or nuclear waste transmutation $[8,9]$. The need for a better theoretical description of spallation reactions has emerged. Furthermore the theoretical investigations in the past were rather fragmentary. First, the activity has centered on neutron double differential cross-sections at some energy and for a few targets, only. It is more and more evident that, besides having a good theoretical tool, the scientists in charge of these projects need to have global information on the properties of the spallation reactions. Just to take an example, it is of crucial importance to know the total neutron multiplicity. To our knowledge the latter has not been measured, at least directly, except for the recent and partial measurements of Refs. [10,11]. Furthermore, theoretically, it would be interesting to know how this quantity (and some other ones) vary with the parameters of the collision. We want to investigate these questions within the INC+evaporation model that we developed during these last years. In fact, we want to make a general survey of the results of this model in the $\mathrm{GeV}$ range. We will not put the emphasis on fine and detailed theoretical predictions (like double differential cross-sections, correlations, ...) nor on their comparison with experimental data (see Ref. [5] for this purpose). Instead, we will focus on the dependence of global properties (like particle multiplicities) with incident energy, target mass and impact parameter. We will also split the yields in INC and evaporation components, when this is illuminating the discussion. As far as we know, only fragmentary accounts of these results exist in the literature, except perhaps for the old work of Ref. [12].

We used the standard Liège INC model [6,5] supplemented by the Dresner evaporationfission model [13]. We recently showed that this model provides a generally good agreement with benchmark measurements of neutron double differential cross-sections and even of residue mass spectrum [5]. The paper is organized as follows. In Section 2 we briefly recall the main ingredients of the model. Section 3 contains the central part of our results, namely the average neutron, proton, pion, ... multiplicities, expressed as functions of incident energy, of target mass and of impact parameter. The respective contributions of the INC and evaporation steps are given. The excitation energy and mass of the remnant after cascade are also examined. Section 4 is devoted to the study of the evolution of the representative point of the system in the excitation energy-mass and charge-mass planes. In Section 5 we show how to split the neutron differential cross- 
section into several components and we analyse, in some typical cases, the target mass dependence of these components. All the investigations in Sections 3-5 refer mainly to proton-induced reactions, but we will also present the most representative results for neutron and deuteron-induced reactions. Finally, Section 6 contains a discussion of the results and our conclusion.

\section{The INC+evaporation model}

The standard Liège INC model for nucleon-induced interactions was first described in Ref. [6]. Minor improvements has been introduced in Refs. [14,15,5]. An exhaustive account can be found in Ref. [5]. It is sufficient here to recall that the collision process is described as a time-ordered sequence of binary collisions (and decays) occurring as in free-space (except for Pauli blocking) between classical nucleons feeling a constant potential well inside the nuclear volume. At some time $t_{\text {fin }}$, the cascade is stopped and the mass, charge and excitation energy of the remnant (defined as the nucleons remaining inside the nuclear volume) are evaluated and used as input of the evaporation-fission code of Ref. [13]. In the latter, light particle evaporation is basically assumed to proceed according to the Weisskopf-Ewing model [16] and the evaporation-fission competition is described following Atchison's formulation. Details, including choice of parameters, are contained in Refs. [17,18]. It is sufficient here to say that the fission probability is given by the Bohr-Wheeler formula [19] and that the competition is present at all stages of the de-excitation process.

Let us say a few words about the value of $t_{\text {fin }}$. The energy of the system can be split into several components, which satisfy the following relation:

$$
T_{0}=\sum_{j=1}^{N_{e j}} \overline{T_{j}}+\sum_{l=1}^{N_{\pi}} \epsilon_{l}+E^{*}+S,
$$

where $T_{0}$ is the incident particle kinetic energy, the first term on the r.h.s. is the kinetic energy of the ejectiles, the second term the energy carried by the pions, the third term the target remnant excitation energy and the last term the difference between the binding energy of the target and the one of the remnant. The bar above the symbol $T_{j}$ indicates that the extra mass energy (with respect to nucleons) should be added for $\Delta$-particles. Let us stress that relation (2.1) holds at any time. A careful analysis of the time evolution of the INC process is contained in Ref. [15]. It shows that the target excitation energy $E^{*}$ raises rapidly when the incident particle penetrates the target, reaches a maximum after a few $\mathrm{fm} / \mathrm{c}$ and decreases first rather quickly (by emission of fast particles). After some time span, the excitation energy decreases further at a much slower rate, akin to an evaporation process. The change of slope in $E^{*}(t)$ is correlated with similar changes in the time variation of many other quantities. Accordingly, we define $t_{\text {fin }}$ as the value corresponding to these changes, and more precisely to the crossing of the two straight lines, characterizing the two regimes, in a semi-logarithmic plot of $E^{*}(t)$. 

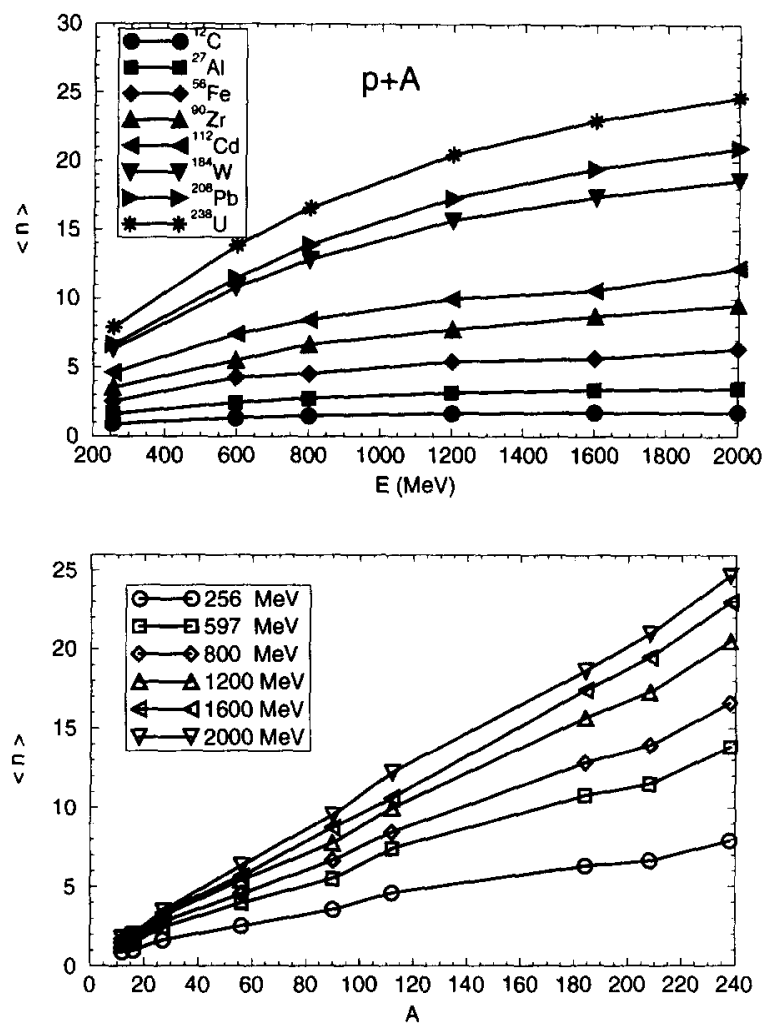

Fig. 1. Average neutron multiplicity versus the incident energy for proton-induced reactions on various targets (upper part) and versus target mass number for various incident energies (lower part).

It is smoothly depending upon target mass, incident energy and impact parameter. For example, $t_{\text {fin }} \approx 25 \mathrm{fm} / c$ for $\mathrm{p}(1 \mathrm{GeV})+\mathrm{Pb}$ central collisions. It is roughly proportional to $A^{1 / 3}$ and only slightly dependent upon the energy in the $0.4-1.2 \mathrm{GeV}$ range.

We also made a few calculations for deuteron-induced reactions. The model is exactly the same except that the projectile is constructed by choosing randomly the relative coordinate and momentum of the neutron-proton system according to Gaussian laws, the widths of which are consistent with known properties of the deuteron. The centre of mass of the projectile is then boosted with the appropriate velocity and impact parameter.

\section{Average properties}

\subsection{Proton induced reactions}

We first discuss the average (over events and impact parameters) neutron multiplicity. This quantity is shown in Fig. 1 for various systems and for different values of the incident proton kinetic energy $E$. In each case (and this also holds for any result 


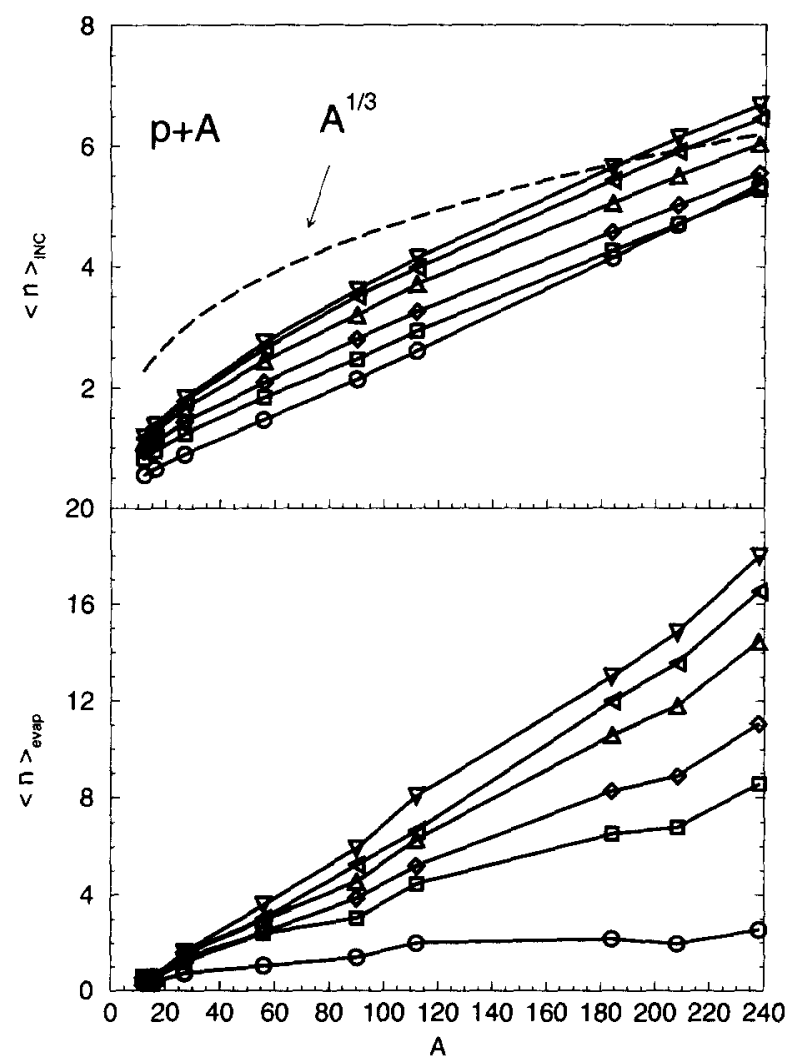

Fig. 2. Average neutron multiplicity in proton-induced reactions, split into cascade (upper part) and evaporation components (lower part). Open symbols refer to the values of the incident energy, with the same convention as in Fig. 1. The dashed line corresponds to the $A^{1 / 3}$ function.

shown in this paper) the average is made on $10^{5}$ events. This guarantees a statistical accuracy better than one percent for any particle multiplicity. Fig. 1 shows that the neutron multiplicity is roughly a linear function of the target mass $A$ and is slowly increasing with incident energy. As a rule of thumb, the average number of neutrons is approximately given by

$$
\langle n\rangle=(0.0803+0.0336 \ln E) A,
$$

where $E$ is the incident proton energy, expressed in $\mathrm{GeV}$, and $A$ is the target mass number. This formula gives a $10 \%$ accuracy or better for $A \gtrsim 40$. The neutron yield is split into cascade and evaporation contributions in Fig. 2 (for the moment we include in the latter contribution pre- as well as post-fission neutrons when fission occurs). Grossly speaking, the latter is more important than the former, by a factor 2 for $E>1 \mathrm{GeV}$. At low incident energy, its importance is reduced. The cascade contribution is roughly a linear function of the target mass number and only weakly dependent upon the energy. This strongly suggests that the most important parameter is the number of primary 


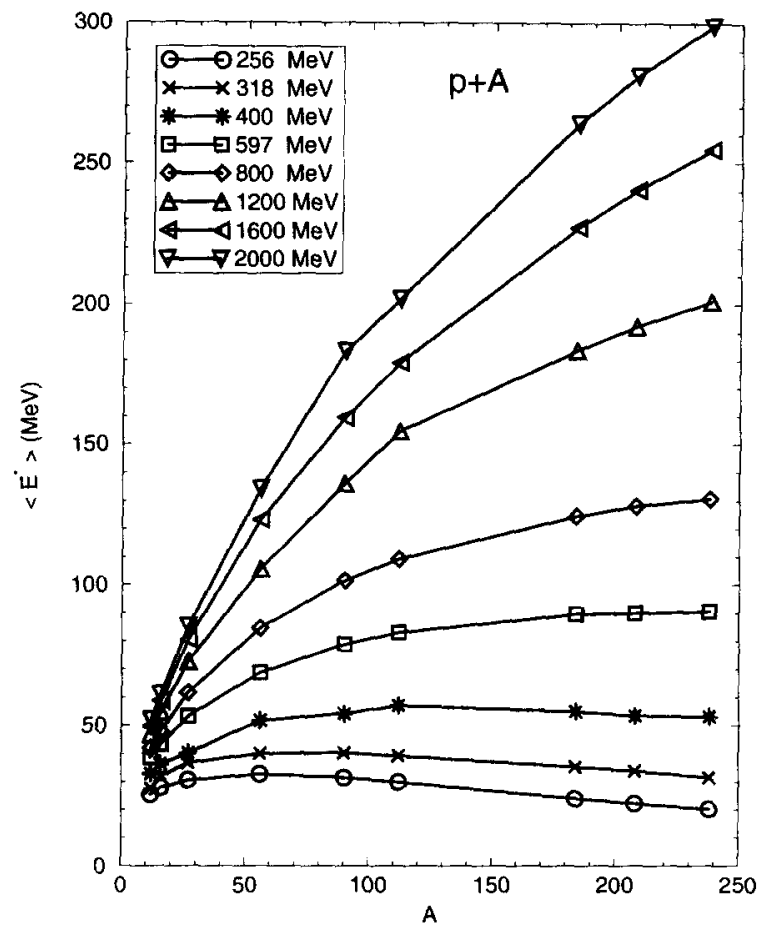

Fig. 3. Target remnant excitation energy at the end of the cascade stage in proton-induced reactions at several incident energies, as a function of the target mass number $A$.

collisions, i.e. the collisions made by the incident particle or by the leading particle. ${ }^{1}$ This quantity does not change strongly with incident energy, since the nucleon-nucleon cross-section is not varying dramatically in the energy range considered here. Fig. 2 shows that, roughly speaking, the multiplicity of the neutrons produced in the cascade stage is close to the $A^{1 / 3}$ law, which is followed by the number of primary collisions [6]. However, secondary collisions are playing some role. They are expected to increase with $A$ faster than the primary collisions. This is responsible for the almost linear variation of the cascade neutron multiplicity with $A$ (see Fig. 2)

The multiplicity of evaporated neutrons is almost proportional to the target mass number and does depend more upon the incident proton energy. The linear dependence upon $A$ is somewhat accidental, as we explain below. But before, let us discuss the excitation energy $E^{*}$ left in the remnant after the cascade. It is given in Fig. 3. Its non-trivial variation needs some comments. First, one has to realize that the average excitation energy is a small fraction of the available energy. In fact, most of the latter is carried away by the final kinetic energy of the projectile (or of the leading particle) and by the kinetic energy of the ejected particles. Fig. 3 shows that $E^{*}$ is increasing with the incident energy, but less than linearly. For light target, the excitation energy is

\footnotetext{
I The incident particle is not always the most energetic one, because of charge exchange reactions among other reasons. See Ref. [6] for a detailed discussion.
} 
Table 1

Values of the parameters $c$ and $d$ for the fit (Eq. (3.2)) for the excitation energy of the remnant

\begin{tabular}{ccc}
\hline Incident energy $(\mathrm{MeV})$ & $c$ & $d$ \\
\hline 256 & 35.848 & -1.068 \\
597 & 19.963 & -0.712 \\
800 & 17.552 & -0.620 \\
1200 & 14.385 & -0.513 \\
1600 & 12.999 & -0.451 \\
2000 & 12.082 & -0.409 \\
\hline
\end{tabular}

quite small, basically because the number of collisions made by the incident particle is always low. For heavier and heavier targets, there are more and more collisions and the energy deposition increases (for a detailed discussion, see Ref. [6]). Note however that the increase with $A$ at given incident energy is less than linear, therefore the quantity $E^{*} / A$ is a decreasing function of $A$, for all cases considered here. The increase of $E^{*}$ with $A$ is less and less pronounced as the incident energy is decreasing, as can be shown in Fig. 3. At the lowest incident energy considered here, the excitation energy is even slightly decreasing with increasing $A$ for $A \gtrsim 50$. We think this is due to the fact that the cascade is lasting for a longer time in heavier targets and thus a larger number of moderately energetic particles can escape. The numerical values of $E^{*}$ can be fitted by the formula

$$
E^{*} / A=c A^{d}
$$

with $c$ and $d$ given numerically in Table 1 . In this formula, $A$ is the initial target mass number. Now, let us come back to the evaporated neutrons. Their number (disregarding competition with other decay channels) can be given in first approximation by

$$
\langle n\rangle_{\text {evap }} \approx \frac{E^{*}}{S_{\mathrm{n}}+3 / 2 T} \approx A \frac{E^{*} / A}{S_{\mathrm{n}}+3 / 2 \sqrt{E^{*} \xi / A_{\text {rem }}}} .
$$

In this equation, $S_{\mathrm{n}}$ is the average neutron separation energy, $T$ the average temperature, $A_{\text {rem }}$ is the remnant mass and $\xi=A / a, a$ being the level density parameter for a nucleus of mass number ${ }^{2} A$. As shown in Fig. $2, A_{\text {rem }}$ is only a few percent smaller than the target mass. Therefore, in Eq. (3.2) and similar ones, we can replace $A_{\text {rem }}$ by $A$ for studying semi-quantitative features. The quantity $S_{\mathrm{n}}$ being slightly decreasing with $A_{\text {rem }}$, for large remnant masses $\left(A_{\text {rem }}>60\right)$, the denominator of the fraction in the previous equation is a smoothly decreasing function of $A$. This variation more or less compensates the slight decrease of $E^{*} / A$ with $A$, yielding an almost linear dependence of $\langle n\rangle_{\text {evap }}$ upon the target mass.

Formula (3.3) helps to understand that the neutron multiplicity is very slowly varying with energy above $1 \mathrm{GeV}$. Indeed with $\xi \approx 8$ and $A_{\text {rem }} \approx A$, one has

\footnotetext{
${ }^{2}$ The factor $3 / 2$ should be replaced by 2 if, as often the case, the so-called surface evaporation model is used rather than the standard Weisskopf-Ewing model [16]. On the other hand, in a chain of neutron emissions, the temperature $T$ is decreasing. A good compromise consists in keeping the factor $3 / 2$, in first approximation.
} 
Table 2

Numerical values of the coefficients entering parametrization (Eq. (3.5))

\begin{tabular}{cllll}
\hline$E(\mathrm{MeV})$ & $a_{1}$ & $a_{2}$ & $b_{1}$ & $b_{2}$ \\
\hline 256 & 0.021 & 0.2967 & 0.0638 & 0.680 \\
597 & 0.019 & 0.6854 & 0.0410 & 0.975 \\
800 & 0.0198 & 0.9090 & 0.0399 & 1.017 \\
1200 & 0.0218 & 1.0372 & 0.032 & 1.1092 \\
1600 & 0.0231 & 1.127 & 0.0291 & 1.155 \\
2000 & 0.024 & 1.1533 & 0.0276 & 1.189 \\
\hline
\end{tabular}

$$
\langle n\rangle_{\text {evap }} \approx \frac{A}{12} \frac{E^{*} / A}{S_{\mathrm{n}} / 12+\sqrt{E^{*} / 8 A}} .
$$

For incident proton energy larger than $\sim 1 \mathrm{GeV}$, the numerical values of $E^{*} / A$ are such that the last fraction is of the order of unity, Therefore $\langle n\rangle=A / 12$ provides a crude approximation of the total neutron multiplicity, as the cascade neutrons are much less numerous. Inspection of Fig. 1 shows that this simple approximation is surprisingly good above $1 \mathrm{GeV}$.

The variation of cascade and evaporation neutron components can be summarized in the following parametrizations

$$
\begin{aligned}
\langle n\rangle & =\langle n\rangle_{\mathrm{INC}}+\langle n\rangle_{\text {evap }} \\
& =\left(a_{1} A+a_{2}\right)+\left(b_{1} A^{b_{2}}\right) .
\end{aligned}
$$

The numerical values of the parameters $a_{1}, a_{2}, b_{1}$ and $b_{2}$ for different values of the incident energy are given in Table 2. Note that $a_{1}$ is always close to 0.02 and $b_{2}$ is always close to unity, except at the lowest incident energy.

In conclusion, the almost linear $A$-dependence of the total neutron yield is largely due to the dominance of evaporation and to the linear dependence of the neutron evaporation yield, which in turn results from the conspiration of the decreases (with $A$ ) of $E^{*} / A$ and of the mean neutron energy. The almost logarithmic energy dependence in Eq. (3.1) is rather accidental and has no precise meaning.

As stated above, the multiplicity $\langle n\rangle_{\text {evap }}$ can be divided into two parts: the multiplicity of neutrons emitted by the remnant, either before complete cool down or before fission if the latter occurs, and the multiplicity of neutrons emitted by the fission fragments. For targets lighter than $\mathrm{Pb}$, the second component is vanishingly small. To give an idea, this quantity amounts to 0.75 and 1.21 in the $\mathrm{p}+\mathrm{Pb}$ system at 800 and $1600 \mathrm{MeV}$, respectively. However, it increases drastically for heavier targets, with the fissility parameter. For the $U$ target, it reaches 6.44 and 9.92 at 800 and $1600 \mathrm{MeV}$, respectively. Apparently, the number of post-fission neutrons is slightly overestimated with respect to the measurements of Hilscher et al. [20]. In any case, the quantity $\langle n\rangle_{\text {evap }}$ is not very sensitive to the fission probability. In fact, we did some illustrative calculations suppressing fission: the results of Fig. $2 \mathrm{~b}$ are practically not changed, except for the $\mathrm{U}$ points which are lowered by one or two units. Neutrons are thus slightly more efficiently produced 
by fission: this is signalled by the fact that the $\mathrm{U}$ points are lying slightly higher than the systematics shown by the other points in Fig. $2 b$.

It is interesting to say a few words about the comparison with the work of Ref. [21]. In the latter, the most extensive measurements of neutron double differential cross-sections in proton-induced reactions (at $590 \mathrm{MeV}$ ) [25] have been exploited to estimate neutron multiplicities. The cross-sections at several angles and for a large domain of neutron energy have been parametrized. Extrapolation to all angles and all energies and estimated total reaction cross-sections are used. Although the extracted numbers are certainly to be considered as crude estimates (presumably an error of one unit is to be considered), we used them to compare with our results. For $\mathrm{Al}, \mathrm{Fe}, \mathrm{Nb}, \mathrm{In}, \mathrm{Ta}, \mathrm{Pb}$ and $\mathrm{U}$ targets, the estimated neutron multiplicity (using formula (8) of Ref. [21]) is 3.55, 5.6, 7.7, 8.9, $11.8,12.8$ and 13.9, respectively. Our predictions are: $2.5,3.9,5.4,7.6,11.0,11.6$ and 13.8. They are somewhat lower for light and medium heavy targets. For this reason, a $A^{2 / 3}$ dependence is recommended in Ref. [21], instead of the linear law embodied by Eq. (3.1). For $A \gtrsim 110$, the numbers of Ref. [21] and our results are in reasonable agreement.

Let us turn to proton emission. The situation is basically reversed, compared to neutrons: protons are mainly emitted in the cascade stage. The contribution of the latter to the average proton multiplicity is given in Fig. 4. For light targets, the proton multiplicity is about the same as the neutron multiplicity, but it increases more slowly with target mass. It is instructive to look at the $n / p$ ratio in the cascade stage (lower part of Fig. 4). For light targets, this ratio is below unity, because the incident proton is going out of a charge symmetric target and thus adds to equal numbers of ejected neutrons and protons. The situation is reversed, for heavy targets. The $n / p$ ratio of the outgoing particles overtakes the $n / p$ ratio of the target. The principal reason is the presence of the increasing Coulomb barrier. This effect is enhanced for small incident energies. There are two reasons for this: (i) outgoing particles are less energetic and therefore has less chance to overcome the barrier (ii) at low energy, the np cross-section is larger than the pp cross-section and therefore the incident proton disturbs the neutrons slightly more than the protons.

The evaporation contribution to proton multiplicity is displayed in Fig. 5, along with the multiplicity of light composites which, in our model, are produced in the evaporation stage only. The incident energy and target mass dependencies are more complicated, although some systematic trends are visible. They are rather standard consequences of the Weisskopf-Ewing theory [16] of evaporation. We just illustrate some of them. In this theory, the width for emission of species $i$ is roughly given by

$$
\Gamma_{i} \approx \frac{2 m_{i} \sigma(i)}{(2 \pi)^{3} \hbar^{2}} \frac{\xi E^{*}}{A} \exp \left(-\frac{S_{i}+B_{i}}{\sqrt{\xi E^{*} / A}}\right),
$$

where $m_{i}$ is the mass of particle $i, \sigma(i)$ its capture cross-section, $S_{i}$ its separation energy and $B_{i}$, the height of the corresponding Coulomb barrier. This equation is obtained after liberal use of the exponential law for the level density. In the proton-nucleus reactions 


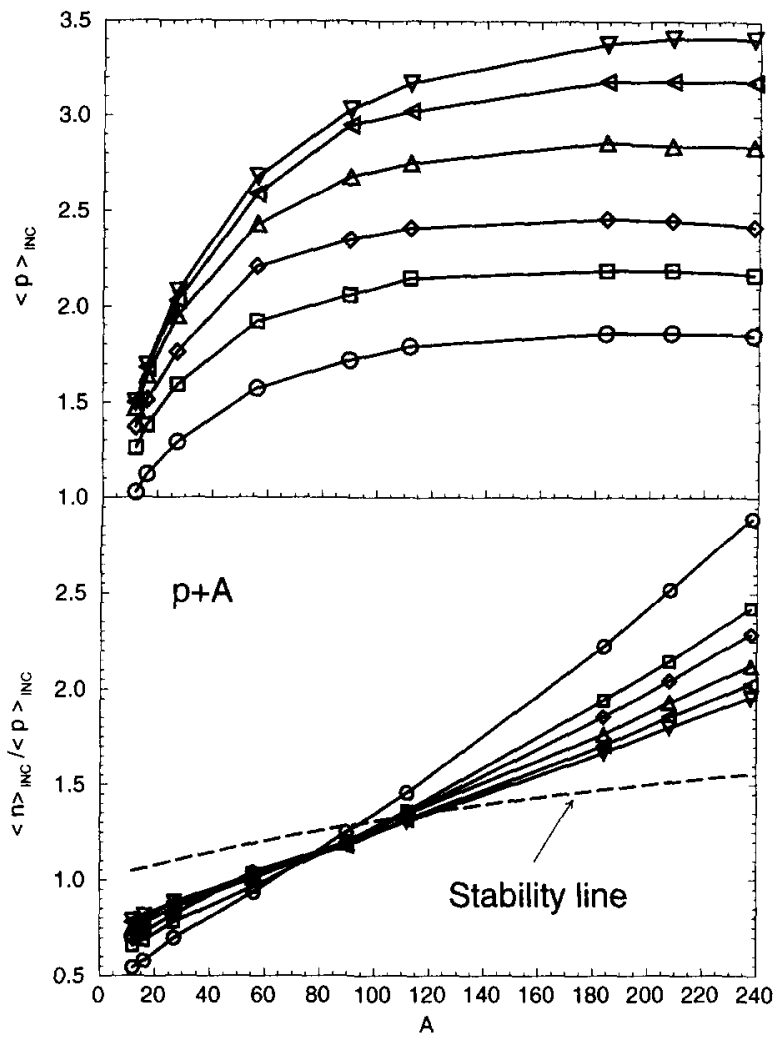

Fig. 4. Average multiplicity of protons emitted in the cascade stage of proton-induced reactions, versus target mass number for various incident energies (upper part). Ratio of average neutron multiplicity on average proton multiplicity for the same conditions (lower part). The dashed line represents the neutron to proton ratio for the stability line. Same convention as in Fig. 1 for open symbols.

that we investigate, the $E^{*} / A$ ratio is moderately dependent upon the incident energy for light targets, and is increasing much more with incident energy for heavy targets (see Fig. 3). Consequently, the particle yields are almost independent of the incident energy for light targets, whereas it becomes more and more important as the incident energy increases for heavy targets. This is corroborated by Fig. 5. The general behaviour of the proton yield with respect to variation of the target mass can be understood as follows. The decrease for $A \gtrsim 60$ is basically due to the increase of the Coulomb barrier. Of course, the larger the value of $E^{*} / A$, the smaller the decrease is; that is why the yield decreases less rapidly in the upper part of Fig. 5 than in the lower part. For small values of $A$, the barrier is not so much important, and the decrease of the yield with decreasing $A$ is mainly due to the decrease of $\sigma(i)$, but competition with $\alpha$ emission is also playing some role. The $\alpha$ yield can also be understood as follows. For heavier and heavier targets, the Coulomb barrier is increasing, but the separation energy is decreasing, becoming even negative. Therefore, $\alpha$-particle production is more and more favoured compared to proton emission. For light nuclei, the separation energy of an $\alpha$-particle is also becoming very small (at least for some of them), and the $\alpha$-particle 


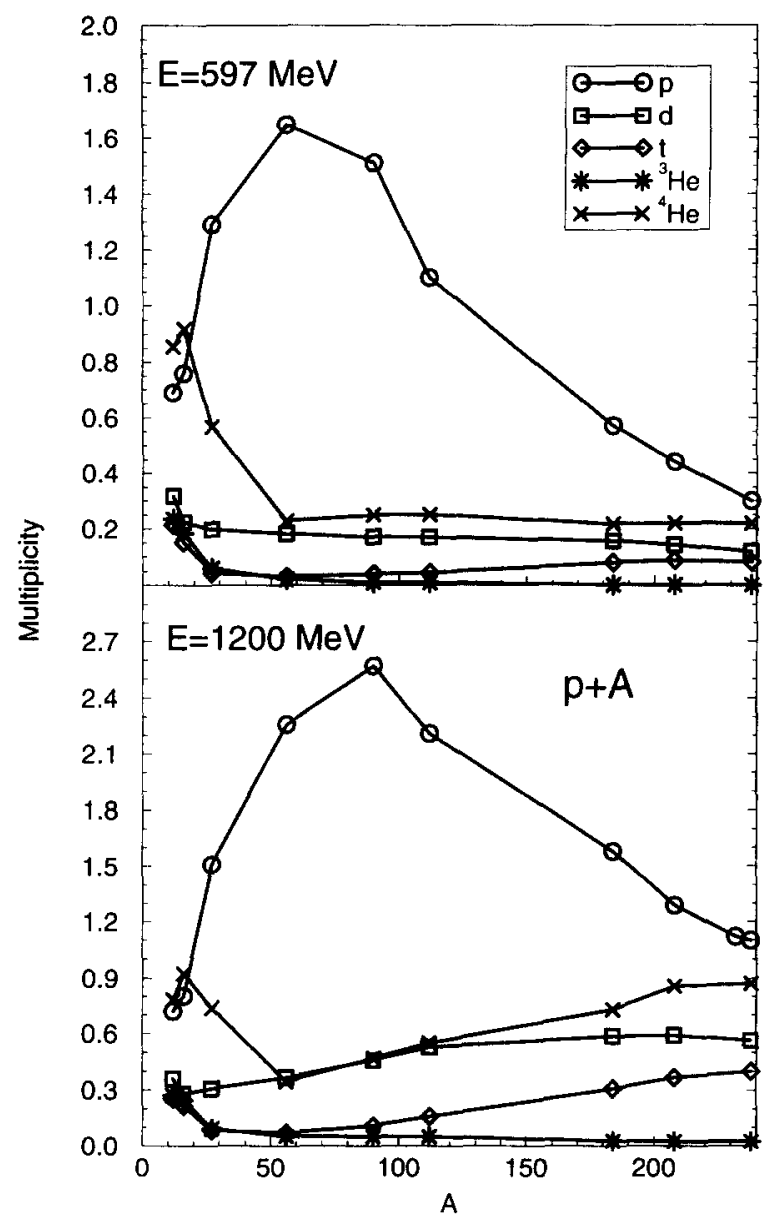

Fig. 5. Average multiplicity of light charged particles emitted during the evaporation stage in proton-induced reactions, versus target mass number $A$ for the two indicated values of the incident proton energy.

is again favoured, compared to the proton emission. The trends for the other particle emission can be understood similarly.

Let us just say a few words about pion production (which is due to the cascade stage). Pion multiplicities in $\mathrm{p}(800 \mathrm{MeV})+A$ reactions are shown in Fig. 6. They are rather low. For small $A$, the calculated values are close to the expectation, based on the single-scattering limit

$$
\begin{aligned}
\left\langle\pi^{+}\right\rangle & =\left(Z \sigma_{\mathrm{pp} \rightarrow \pi^{+}}+N \sigma_{\mathrm{pn} \rightarrow \pi^{1}}\right) / \sigma_{r}^{\mathrm{tot}}, \\
\left\langle\pi^{0}\right\rangle & =\left(Z \sigma_{\mathrm{pp} \rightarrow \pi^{0}}+N \sigma_{\mathrm{pn} \rightarrow \pi^{0}}\right) / \sigma_{r}^{\mathrm{tot}}, \\
\left\langle\pi^{-}\right\rangle & =N \sigma_{\mathrm{pn} \rightarrow \pi^{-}} / \sigma_{r}^{\mathrm{tot}},
\end{aligned}
$$

with $\sigma_{r}^{\text {tot }}$ being the total reaction cross-section, and the isobar model, establishing simple relations between all cross-sections entering Eq. (3.7). For heavy targets, due to more and more important secondary collisions, the charge distribution of the pion system, 


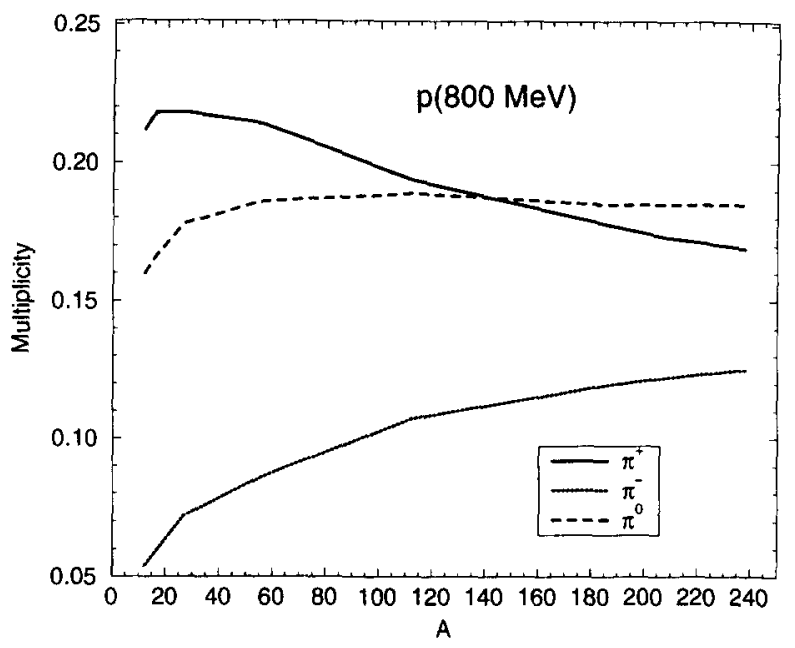

Fig. 6. Average pion multiplicities for the three charge states in proton-induced reactions at $800 \mathrm{MeV}$ incident energy, as functions of the target mass number $A$.

which initially contains more positive charges than negative charges tends to equilibrate with the one of the nucleons, which contain more neutrons than protons: the $\pi^{+}$multiplicity is decreasing and the $\pi^{-}$multiplicity is increasing. The $\pi^{0}$ multiplicity deviates somehow from this trend. It overtakes the $\pi^{+}$multiplicity, because the np inelastic cross-section is slightly larger than in the isobar model and because charge exchange between $\Delta$ 's and nucleon does not follow isobar symmetry in our cascade model. This favours $\pi^{0}$ production. Charged pion multiplicities for all incident energies are given in Fig. 7. They are plotted against variables, which are borrowed from the work of Ref. [22]. According to the latter, the constancy of the $\pi^{+}$multiplicity with respect to the first variable would be an indication of the production in the first collisions and the constancy of the $\pi^{-}$multiplicity with respect to the second variable would point to some importance of the rescattering. This is more or less the case for our $\pi^{+}$results, in agreement with the comments about Fig. 6. For $\pi^{-}$production, the secondary collisions seem however to be more important than suggested by the recommended variable.

\subsection{Neutron induced reactions}

Most of the results are amazingly similar to those of proton induced reactions, except for some qualitative and obvious differences. As an example, we show in Fig. 8 the calculated neutron multiplicities. They are practically the same as those for protoninduced reactions after addition of one unit, whose relative importance manifests itself for light targets only. The rule of thumb, similar to Eq. (3.1) and valid for $A \gtrsim 60$, takes now the form

$$
\langle n\rangle=(0.0833+0.0317 \ln E) A .
$$



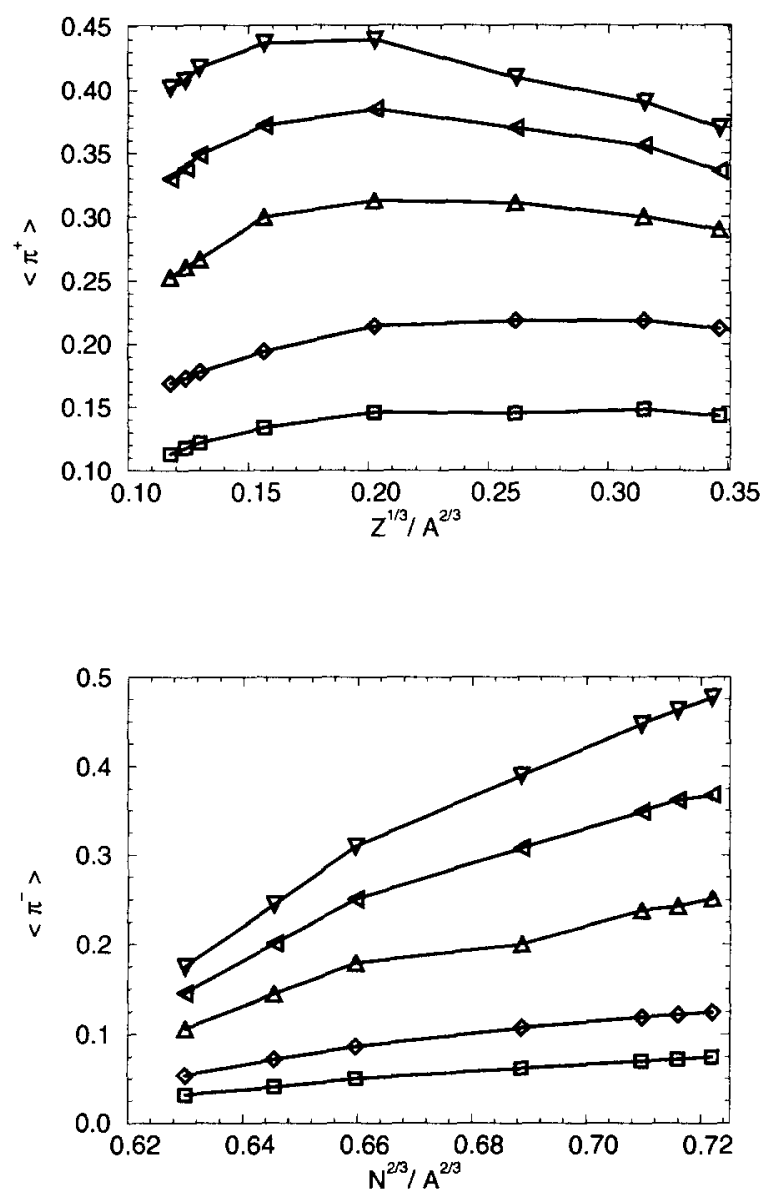

Fig. 7. Average charged pion multiplicities in proton-induced reactions. They are plotted versus the variables taken from Ref. [22]. The symbols $A, N, Z$ stand for the target mass, neutron and proton numbers, respectively. The various lines correspond to various incident energies and the symbols represent targets ranging from ${ }^{12} \mathrm{C}$ to ${ }^{238} \mathrm{U}$. Same convention as in Fig. 1 for the open symbols.

Table 3

Numerical values of the coefficients entering parametrization (Eq. (3.5)) of the average neutron multiplicity in neutron-induced reactions

\begin{tabular}{rllll}
\hline$E(\mathrm{MeV})$ & $a_{1}$ & $a_{2}$ & $b_{1}$ & $b_{2}$ \\
\hline 256 & 0.020 & 0.801 & 0.1055 & 0.595 \\
597 & 0.019 & 1.190 & 0.0500 & 0.942 \\
800 & 0.0198 & 1.331 & 0.0422 & 1.021 \\
1200 & 0.0214 & 1.438 & 0.037 & 1.089 \\
1600 & 0.023 & 1.479 & 0.0342 & 1.1130 \\
2000 & 0.024 & 1.495 & 0.0328 & 1.154 \\
\hline
\end{tabular}



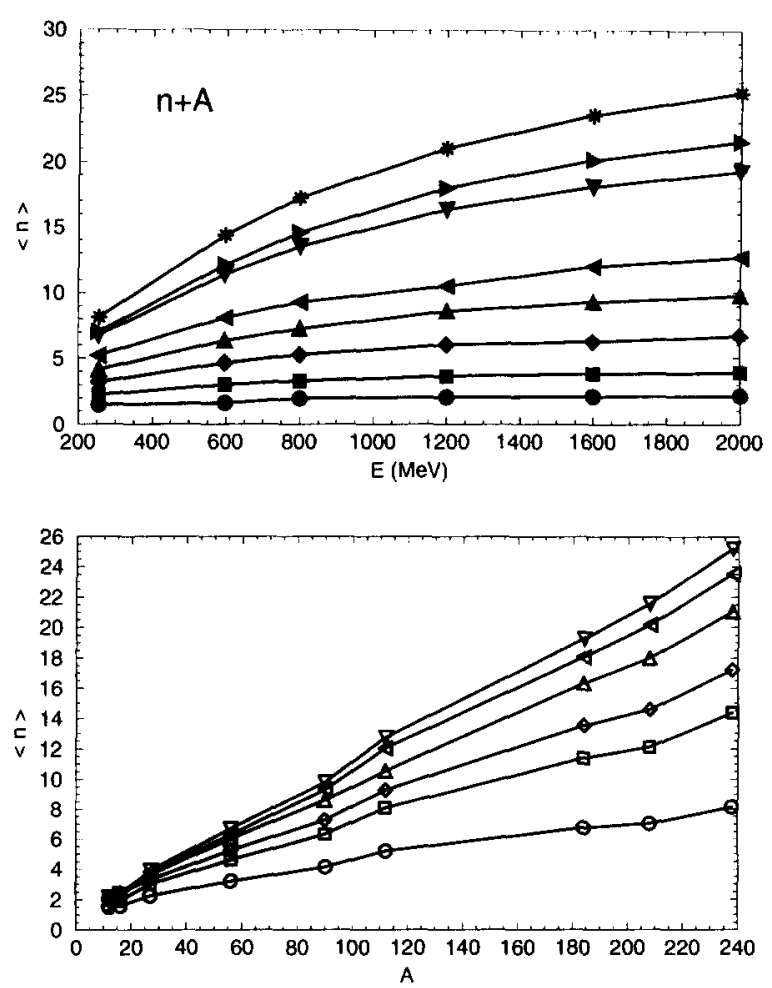

Fig. 8. Average neutron multiplicity versus incident energy for neutron-induced reactions on various targets (upper part) and versus target mass number for various incident energies (lower part). Same convention as in Fig. I for both black and open symbols.

The INC and evaporation components can be fitted by expression (3.5), with parameters given in Table 3. It is amusing to look at the behaviour of the $n / p$ ratio of the ejectiles (Fig. 9): for light targets, it is larger than unity, because of the extra neutron corresponding to the incident particle. The dependence upon the incident energy can be understood as before. The number of ejected cascade nucleons and the excitation energy, are practically the same as in the proton-induced case, especially for heavy targets. The evaporated particle yield is very close to its value for the proton-induced case. Pion multiplicities are given in Fig. 10. As expected, negative pion production is favoured in this case.

\subsection{Impact parameter dependence}

We illustrate in Fig. 11, for the $\mathrm{p}(2000 \mathrm{MeV})+\mathrm{Pb}$ system, the characteristic impact parameter dependence of some calculated quantities. The number of cascade particles (lower part) is decreasing with increasing impact parameter $b$, in a way which resembles a semi-circle law. The latter is expected when the relevant parameter is the length of the path followed by the incident particle. The remnant excitation remains roughly constant up to half maximum value of $b$ and then decreases further. The average remnant angular 


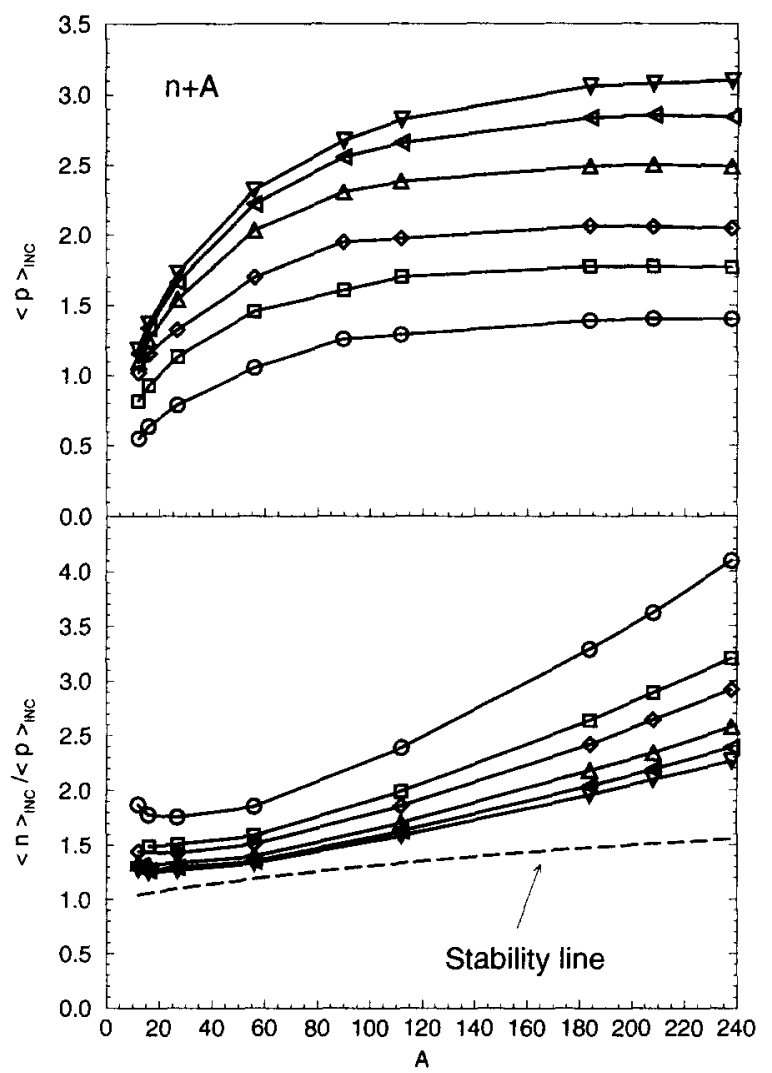

Fig. 9. Same as Fig. 4 for neutron induced reactions. Same convention as in Fig. 1 for open symbols.

momentum is weakly dependent upon the impact parameter. However, it should be realized that, for $b \approx 0$, angular momentum vector is oriented practically at random, event by event, whereas it is more and more aligned with the initial angular momentum for increasing values of $b$. These typical variations are qualitatively the same at all the energies investigated here.

It is of interest to look at the impact parameter splitting of the double differential crosssections. The neutron one is displayed in Fig. 12, for the $p(800 \mathrm{MeV})+\mathrm{Fe}$ system. Only the large impact parameter collisions contribute significantly to the large neutron energy domain ( $\gtrsim 300 \mathrm{MeV})$ at small angles, corresponding more or less to the quasi-elastic and quasi-inelastic regions. Intermediate impact parameters contribute everywhere. Small impact parameters contribute essentially to small neutron energies. It should be noticed that the large impact parameters contribute also importantly to the low energy domain. This presumably comes from the target particles which are the partners of the high energy particles coming out in the quasi-elastic and quasi-inelastic scatterings. 


\section{$n+A$}

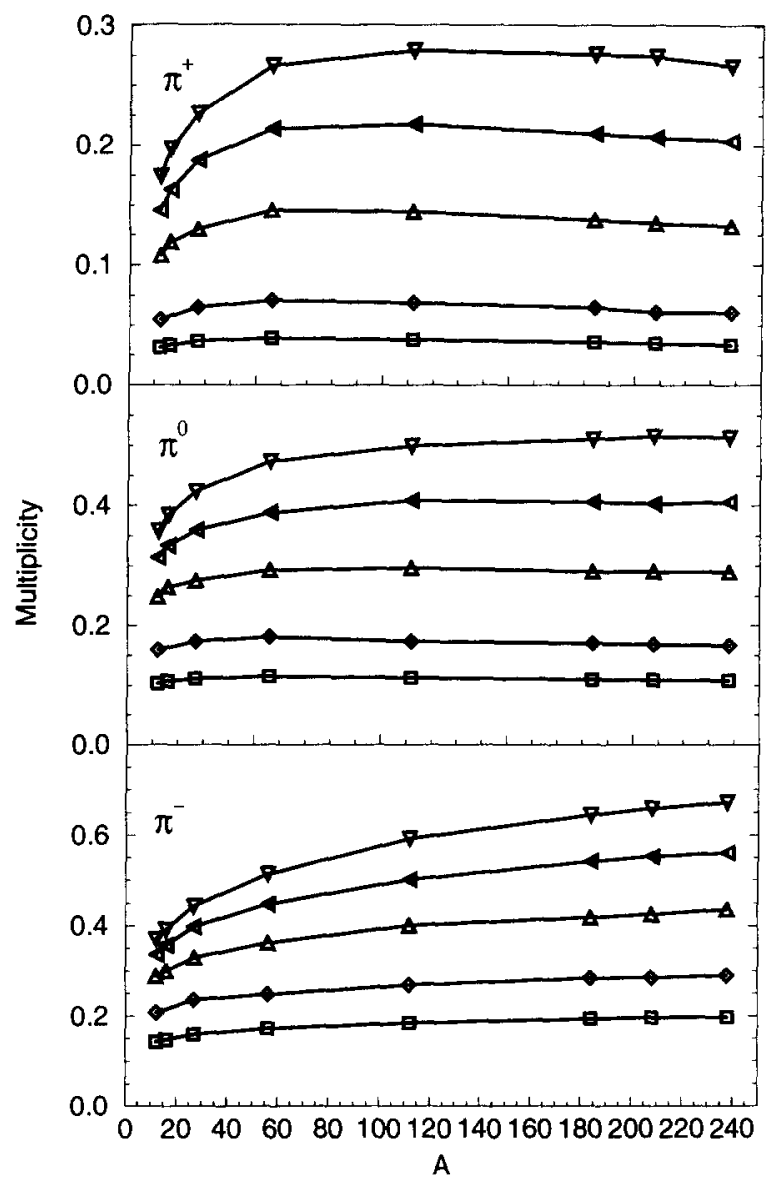

Fig. 10. Average pion multiplicities versus target mass number $A$ for neutron-induced reactions on various targets and at various incident energies. Same convention as in Fig. 1 for open symbols.

\section{Residue mass distribution}

Let us first discuss the mass-charge distribution in the INC+evaporation model. Fig. 13 shows this distribution after the cascade step (the remnants) and after the evaporation step (the residues ${ }^{3}$ ), for the $\mathrm{p}(1000 \mathrm{MeV})+\mathrm{Pb}$ system. On the average, the cascade step drives the representative point of the (compound) system, almost in a parallel direction with the stability line (this corresponds to a $n / p$ ratio of the removed nucleons larger than the one of the target, in accordance to our previous discussion). Note however that the fluctuations are almost as large as the average shift. The evaporation step drives the representative point towards the proton rich side of the stability valley, because this step is dominated by neutron emission. The fission fragments that are represented in Fig. 13

\footnotetext{
${ }^{3}$ This term, which usually designates the target system after $\beta$ and $\gamma$ decays, is used here unconventionally.
} 


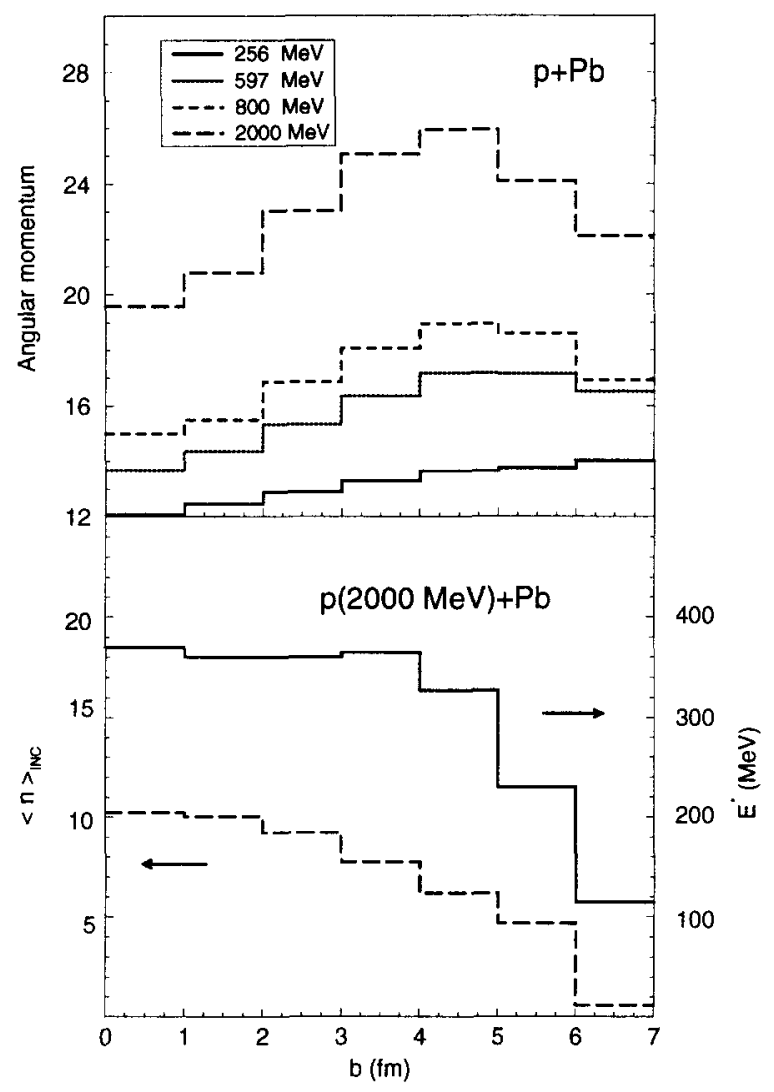

Fig. 11. Impact parameter dependence of various quantities in proton-induced reactions. Upper part: average target remnant angular momentum at the end of the intranuclear cascade stage for various impact parameter bins and at the indicated values of the incident energy. The curves correspond to the indicated incident energies. Lower part: average multiplicity of neutrons emitted in the cascade stage (dashed curve, scale on the left) and target remnant excitation energy at the end of this stage (full curve, scale on the right), for various impact parameter bins in $p(2000 \mathrm{MeV})+\mathrm{Pb}$ reactions.

by the elongated spot between, say $N \sim 30$ and $N \sim 70$. The latter is roughly lying on the stability line and not on the neutron rich side of the valley, as for spontaneous fission. This originates from two reasons. First the remnants which are the best candidates for fission are those which have the largest fissility parameters, i.e. those which are lying the farthest on the proton side of the valley. In Fig. 13a the fissility parameter ranges from 27 to 35, when going from the bottom to the top of the contour plot. Neutron evaporation accentuates this tendency.

The joint mass and excitation energy distribution at the end of the INC step is shown in Fig. 14 for the $\mathrm{p}(1 \mathrm{GeV})+\mathrm{Pb}$ case. As expected, the heaviest remnants have on the average low excitation energy. The average excitation energy is increasing when the remnant mass decreases, although some saturation seems to occur for $A \sim 200$. Finally, let us remind that the average excitation energy is of the order of $150 \mathrm{MeV}$.

The calculated residue mass spectrum is displayed in Fig. 15 for the $\mathrm{p}(1 \mathrm{GeV})+\mathrm{Pb}$ 


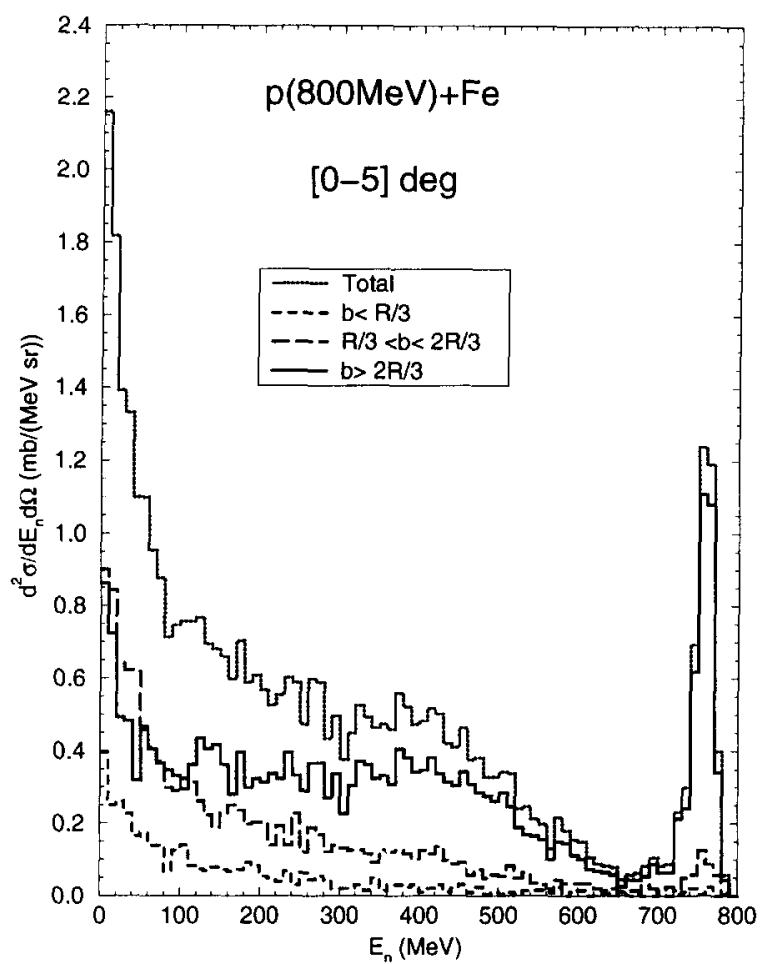

Fig. 12. Splitting of the neutron double differential cross-section (averaged over the $0-5^{\circ}$ angular range) into contributions from different impact parameter bins.

system at several incident energies. The obvious features are the broadening of the so-called spallation peak, the increase of the fission peak and the saturation apparently setting in at high incident energy. They more or less proceed from the variation of the excitation energy with the incident energy. Finer details are noticeable. The fission peak is shifting to lower masses as the incident energy increases. This feature presumably results from the more and more important neutron emission prior to fission. A shallow minimum appears in the spallation peak for incident energy above $1 \mathrm{GeV}$ incident energy. We interpret this as a subtle variation of the fission probability within the masscharge distribution after the INC step. For the masses closest to the target mass, the excitation energy is relatively low and so is the fission probability. For the intermediate mass values inside this distribution, both the fissility and the excitation energy are larger, and therefore the fission probability is increased. For still lower masses, the average excitation energy is still large, but the fissility parameter is decreasing and therefore the fission probability starts to decrease. Let us notice that this behaviour of the mass distribution has been observed in $\mathrm{p}(590 \mathrm{MeV})+{ }^{238} \mathrm{U}$ reactions [23]. We reproduce this behaviour qualitatively but not quantitatively. This may indicate that the fission probability at large excitation energy is underestimated in the Dresner evaporation model [13]. However, it is shown in Ref, [5] that we correctly reproduce the mass spectrum in $\mathrm{p}(800 \mathrm{MeV})+\mathrm{Pb}[23]$ and $\mathrm{p}(1 \mathrm{GeV})+\mathrm{Au}[24]$ reactions. 

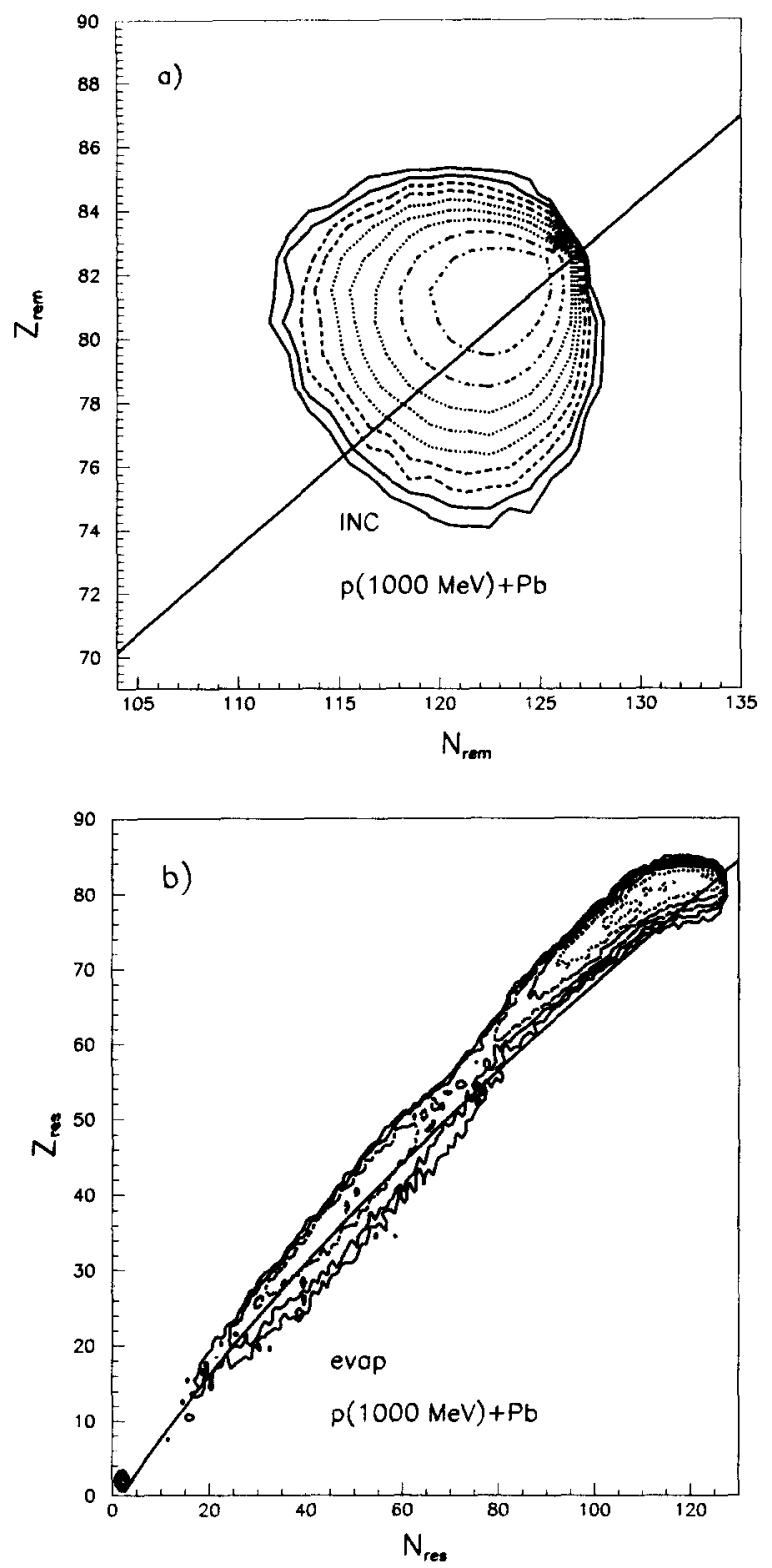

Fig. 13. Target remnant distribution (in the $N-Z$ plane) after the cascade stage (a) and target residue distribution after the evaporation stage (b) in $\mathrm{p}(1000 \mathrm{MeV})+\mathrm{Pb}$ reactions. In (a) the star gives the location of the target plus incident proton system in the $N-Z$ plane. In both (a) and (b) the heavy line represents schematically the stability line. Going from one contour line to the next outer one corresponds to a decrease of a factor $\sim 2.77$ in the yield. 


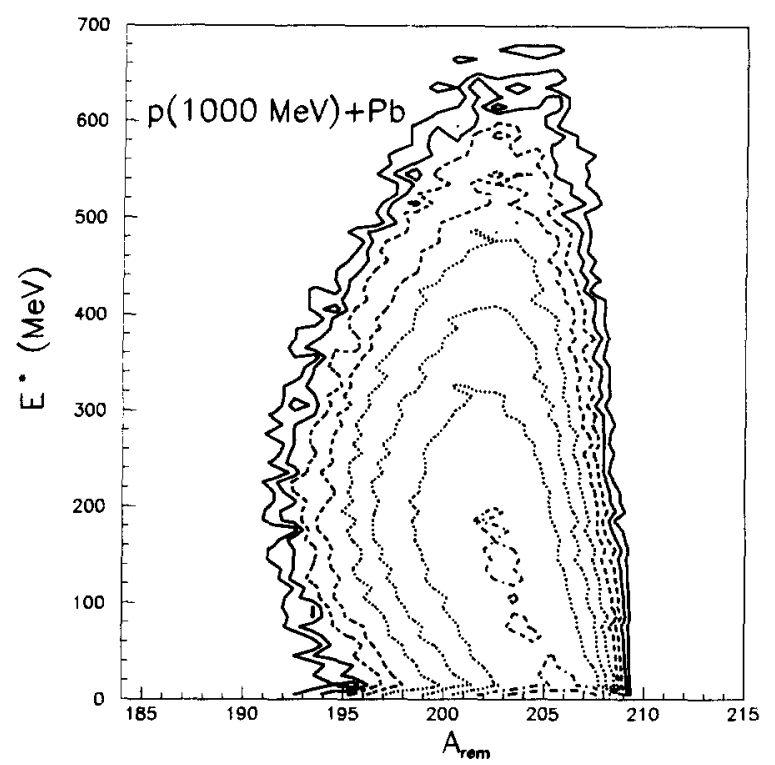

Fig. 14. Contour plot of the target remnant mass-excitation energy distribution at the end of the cascade stage, in $\mathrm{p}(1000 \mathrm{MeV})+\mathrm{Pb}$ reactions. Going from one contour line to the next outer one corresponds to a decrease of a factor $\sim 3.3$ in the yield.

\section{Decomposition of the neutron double differential cross-section}

In the energy range considered in this study, the experimental neutron double differential cross-sections display characteristic variations with neutron energy and angle which suggests a decomposition of the spectra into components that have an obvious physical origin [5] (this is also true to some extent for other particle production cross-sections). The same remark applies, of course, to our calculated cross-sections. We want to perform such a decomposition of the latter, in order to exhibit the dependence of these components with target mass. Furthermore we want to see whether the evaporation and cascade components, as produced in our model, can be recovered in this decomposition. The neutron spectra averaged over the $0-5^{\circ}$ range and over the $140-160^{\circ}$ in p-induced reactions ( see Fig. 16) have been decomposed by fitting them with the following form:

$$
\begin{aligned}
\frac{d^{2} \sigma}{d \Omega d E}= & A_{1} \exp \left(-E / E_{1}\right)+\sum_{i=2}^{3} A_{i} \exp \left(-E / E_{i}\right)+A_{\text {in }} \exp \left(-\left(\frac{E-E_{\text {in }}}{W_{\text {in }}}\right)\right)^{2} \\
& +A_{\mathrm{el}} \exp \left(-\left(\frac{E-E_{\mathrm{el}}}{W_{\mathrm{el}}}\right)\right)^{2} .
\end{aligned}
$$

The first term is expected to describe the evaporation component. The third and fourth terms are standing for the quasi-inelastic and quasi-elastic components, respectively. These correspond to neutrons which are ejected after suffering only one collision, the latter leaving the partner nucleon excited to the $\Delta$ resonance, or being elastic, respectively [5]. Finally the second term is expected to single out the cascade component. 


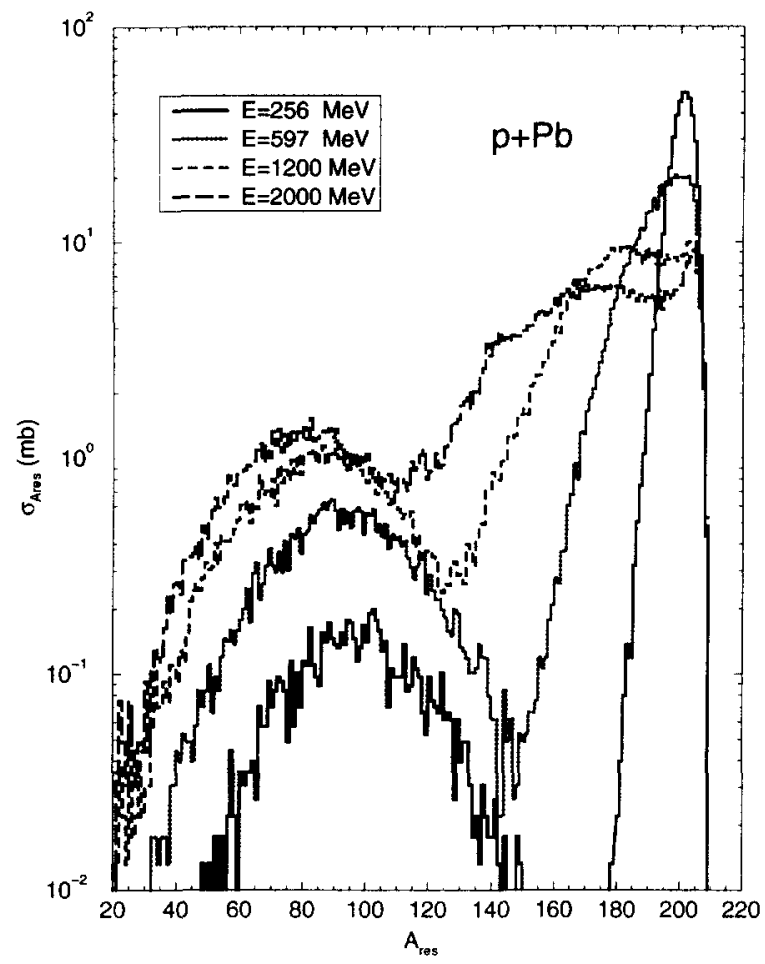

Fig. 15. Cross-sections for production of residue of mass $A_{\text {trs }}$ at the end of the evaporation stage in $\mathrm{p}+\mathrm{Pb}$ reactions at four incident proton energies.

Using a single exponential is not sufficient to achieve a good fit. The quality of the fits is illustrated in Fig. 16 for the Pb target case. Fits of similar quality are obtained for other targets. The parameters of the first term and of the first exponential in the second term have been kept the same at the angles under consideration. No other constraint is imposed. It is interesting to see that the fitting procedure nicely separate the evaporation component and the cascade component, as they are defined in the model. The evaporation component is only slightly underestimated, by a few percent. Therefore one can expect that a similar fitting of the experimental data would be able to isolate the evaporation component with the same accuracy.

The target mass dependence of the various components has been worked out by studying several targets extending from $\mathrm{Al}$ to $\mathrm{Pb}$. We first discuss the results at forward angles. The width of the quasi-elastic peak is practically independent of the target mass and the intensity $A_{\mathrm{el}}$ is proportional to $A^{1 / 3}$. This is rather expected from the fact that the quasi-elastic scattering is possible in peripheral collisions only (as already shown in Fig. 12), because of the probable re-interaction in more central reactions. Assuming a constant band width of the relevant impact parameters would yield this result. The width of the quasi-inelastic component is also practically independent of the target mass, but the intensity ( $A_{\text {inel }}$ ) is proportional to $A^{1 / 2}$. We have no simple explanation for this law. The parameter $A_{1}$ of the evaporation component behaves like $A^{2}$. The width parameter 


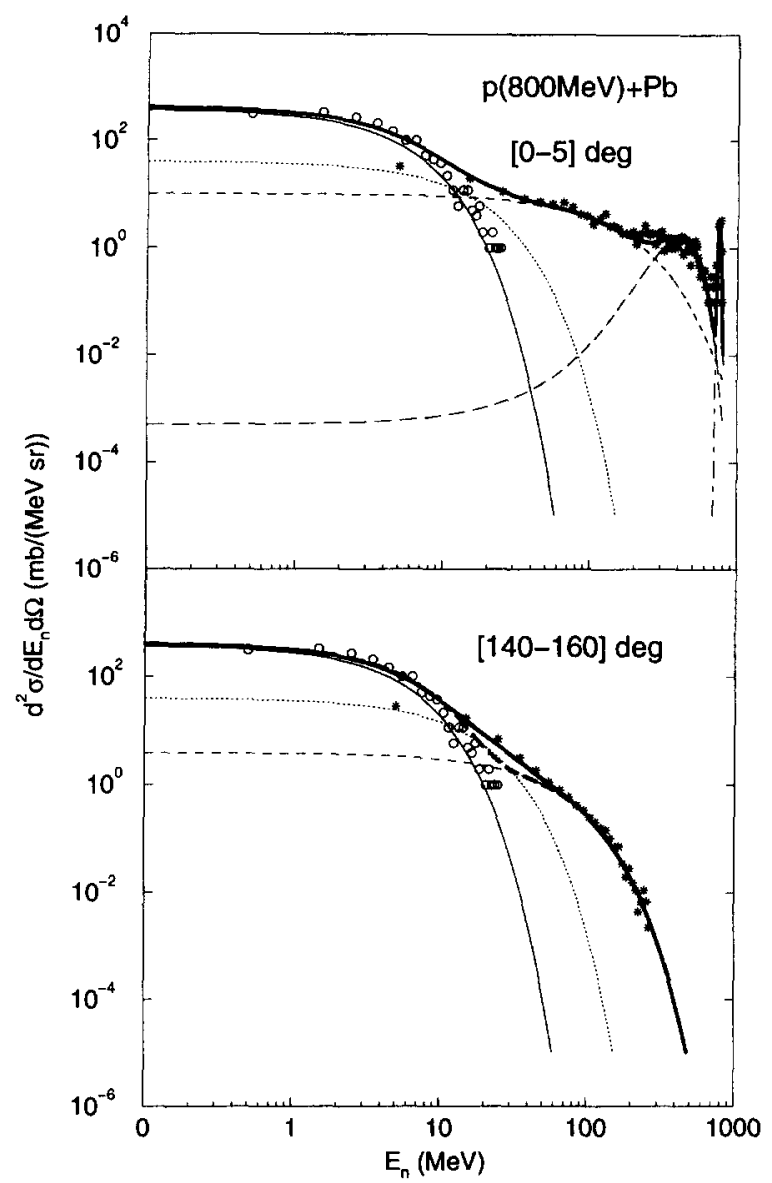

Fig. 16. Neutron double differential cross-sections averaged over angles between $0-5^{\circ}$ (upper part) and $140-160^{\circ}$ (lower part), in $\mathrm{p}(800 \mathrm{MeV})+\mathrm{Pb}$ reactions. The symbols give the results of the INC+evaporation calculation, split into INC (stars) and evaporation (open circles) contributions. The heavy lines represent the fit of these results by formula (5.1) and the thin lines gives the various contributions: first term (full line), second term (dotted and small dashed lines), third term (long dashed line) and fourth term (dot-and-dashed lines) of expression (5.1). In the lower part, the curve in heavy dashes represents the fit when only one exponential in the second term of formula (5.1) is retained.

$E_{1}$ is decreasing slightly with $A$, in keeping with the decreasing value of $E^{*} / A$ with increasing target mass and therefore of the apparent neutron temperature. The product $A_{1} E_{1}$, which is proportional to the neutron evaporation yield, is scaling as $A^{3 / 2}$. This is more or less consistent with the fact the properties of the evaporation neutron multiplicity, that is proportional to $A$ (see Fig. 1), and of the total reaction cross-section, that behaves roughly as $A^{2 / 3}$. The small difference between the two exponents is possibly be due to the fitting procedure. We would like to mention that in Ref. [26], a similar fit is applied to the data for proton-induced reactions and a power law is obtained with a similar value of the exponent. Alike $A$-dependencies are obtained from the fit of the recently measured neutron double differential cross-sections at $0^{\circ}$ on various targets at SATURNE [27]. 
It should be stressed however that these power laws are very well fulfilled for all targets, but $\mathrm{U}$. The corresponding value for the latter overshoots the systematic law by a few percent. This, of course, comes from the large fissility of the target and the extra neutrons released by the (excited) fission products. Finally the typical variation of the "cascade" component is a little bit more complex. The width parameter $E_{2}$ is practically independent of the target mass, whereas the intensity parameter $A_{2}$ behaves like $A^{3 / 2}$. The width parameter $E_{3}$ shows a weak dependence upon $A, \sim A^{0.1}$, to a good precision. The product $A_{3} E_{3}$ is proportional to $A^{0.6}$. These particular dependencies are difficult to interpret. It is noticeable that the importance of the various components (the product of respective amplitude and width parameters) exhibits power laws with increasing values of the exponent of $A$, starting from the largest and going to the lowest neutron energies.

At backward angles, the quasi-elastic and quasi-inelastic components are vanishingly small. The parameters $A_{1}, E_{1}, A_{2}, E_{2}$ have the same values as at forward angles, by construction. The width parameter $E_{3}$ is almost independent of the target mass and the parameter $A_{3}$ is proportional to $A^{0.8}$.

The fact that the cascade component cannot be fitted by a single simple function is underlining the complexity of the process, which cannot probably be reduced to a simple diffusion model for instance. The need for introducing two exponentials is indicated by the lower part of Fig. 16. The broad dashed line correspond to using a single exponential function for the cascade step. At forward angles, a similar fit yield slightly better results, but then the power law in the target mass dependence does not show up so nicely. Finally, it is interesting to note that the amplitude of the first exponential function of the second term in Eq. (5.1), i.e. the product $A_{2} E_{2}$ has approximately the same $A$-dependence as the evaporation component. This reflects the smooth transition between the INC and the evaporation stage in our model and, as far as the observables discussed here are concerned, the absence of a need for introducing an intermediate step (see also Ref. [5] for a discussion of this point).

\section{Deuteron induced reactions}

We have performed the same analysis as above for deuteron-induced reactions. We only single out the most salient features. The calculated total neutron multiplicity is displayed in Fig. 17. It is roughly given by

$$
\langle n\rangle=(0.121+0.0469 \ln E) A .
$$

We stress that in this equation, $E$ represents the incident energy per nucleon (in $\mathrm{GeV}$ ). As before, we split the total multiplicity into cascade and evaporation components, according to the following fitting formula

$$
\langle n\rangle=\langle n\rangle_{\mathrm{INC}}+\langle n\rangle_{\mathrm{evap}} \approx a_{1} A^{a_{2}}+b_{1} A^{b_{2}},
$$

Contrary to the previous cases, a power law is slightly better than a linear form for fitting the cascade component. The numerical values of the coefficients are given in Table 4. 

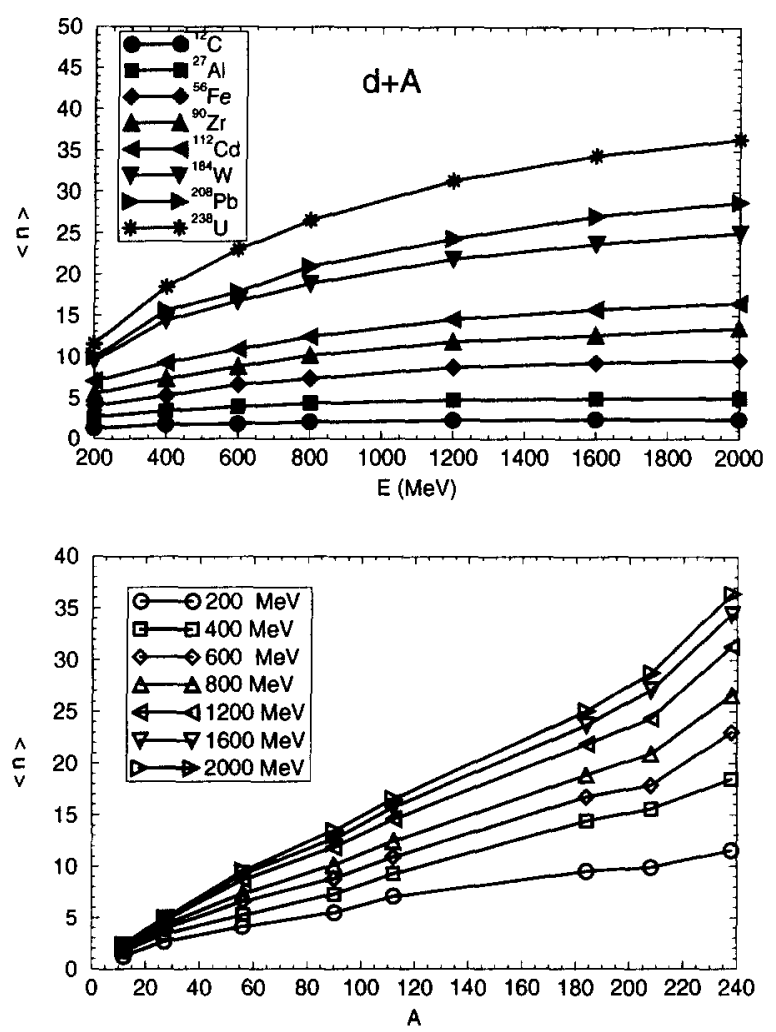

Fig. 17. Average neutron multiplicity versus the incident energy for deuteron-induced reactions on various targets (upper part) and versus target mass number for various incident energies (lower part). Note that $E$ is the incident energy per nucleon.

Table 4

Numerical values of the coefficients entering parametrization (Eq. (6.2)) of average neutron multiplicity in deuteron-induced reactions. $E$ is the incident energy per nucleon

\begin{tabular}{cllll}
\hline$E(\mathrm{MeV})$ & $a_{1}$ & $a_{2}$ & $b_{1}$ & $b_{2}$ \\
\hline 200 & 0.169 & 0.656 & 0.060 & 0.825 \\
400 & 0.24 & 0.578 & 0.033 & 1.08 \\
600 & 0.35 & 0.534 & 0.033 & 1.127 \\
800 & 0.426 & 0.51 & 0.030 & 1.176 \\
1200 & 0.493 & 0.505 & 0.026 & 1.239 \\
1600 & 0.498 & 0.519 & 0.024 & 1.27 \\
2000 & 0.488 & 0.53 & 0.022 & 1.30 \\
\hline
\end{tabular}

For completeness, the two components are given in Fig. 18. It is to be noticed that evaporated neutrons for the $U$ target are slightly more numerous that expected from the systematic trend indicated by the other targets. This of course comes the extra neutrons emitted by the fission fragments. To give an idea, suppressing fission reduces the neutron multiplicity by 1.3 units at an incident energy of $1600 \mathrm{MeV}$ per nucleon. Because of 


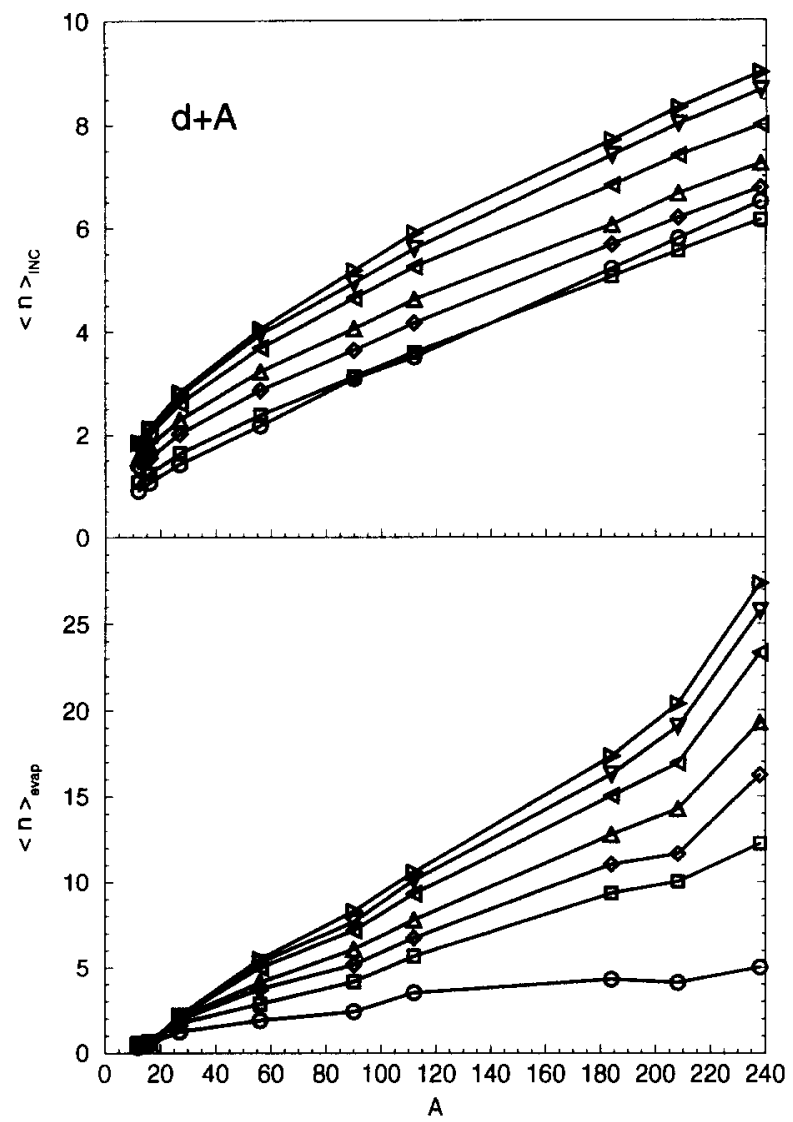

Fig. 18. Average neutron multiplicity for deuteron-induced reactions, as a function of the target mass number for various values of the incident deuteron energy per nucleon. The upper part refers to neutrons emitted in the cascade stage and the lower part, to neutrons emitted in the evaporation stage. Same convention as in Fig. 17 for open symbols.

this "anomaly", formula (6.2) slightly overestimates the neutron yield for the $\mathrm{W}$ and $\mathrm{Pb}$ targets. The excitation energy is given in Fig. 19.

It is interesting to compare the results with those of nucleon-induced reactions. First, the total neutron multiplicity in deuteron-induced reactions is less than the sum of the total neutron multiplicity in proton-induced reactions and of the same quantity in neutron-induced reactions, for the same target and the same incident energy per nucleon. The non-additivity of the cascades initiated by the neutron and the proton contained in the deuteron comes from two facts. First, for peripheral collisions, one of the nucleons may not interact at all, corresponding to a stripping reaction. Second, for more central collisions, the propagation of one of the nucleons "clears the space" seen by the other nucleon. It can be seen from formulae (3.1) and (6.1) that the reduction is the order of 20-30\%. This result can be understood as follows. First, the excitation energy is about twice as large for deuteron-induced reactions as for proton-induced reactions, at the same incident energy per nucleon (compare Figs. 3 and 19). As a result, the mean number of 


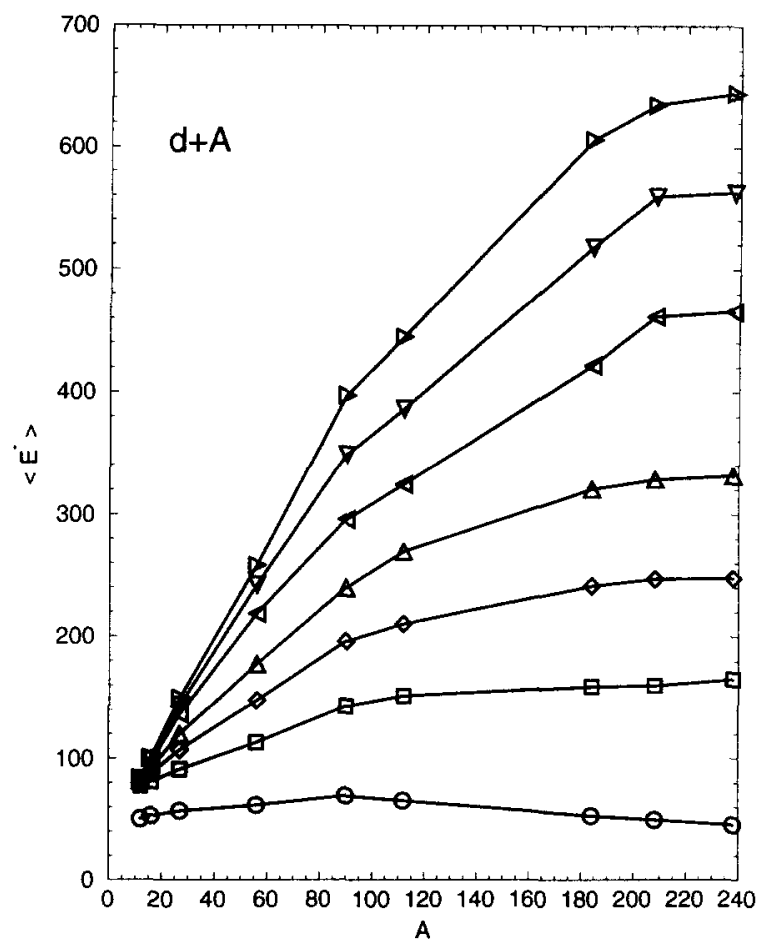

Fig. 19. Target remnant excitation energy at the end of the cascade stage in deuteron-induced reactions at several values of the incident energy per nucleon, as a function of the target mass number $A$. Same convention as in Fig. 17 for open symbols.

evaporative neutrons is less than twice the value for proton-induced reactions, because neutrons emitted at larger excitation energy carry away more energy (see discussion about Eq. (3.3)). Second the number of cascade neutrons in deuteron-induced reactions is also slightly less than twice the number for nucleon-induced reactions.

On the other hand, from the same formulae (3.1) and (6.1), it can be seen that the neutron multiplicity is about the same for proton-induced reactions and deuteron-induced reactions at the same total incident energy (from $400 \mathrm{MeV}$ to $2 \mathrm{GeV}$ ). This is in fact somewhat accidental, as for instance, the energy dependence of the neutron multiplicity, embodied by the coefficients of the parentheses appearing in Eqs. (3.1) and (3.8) are quite different. It turns out that for a total incident energy between $400 \mathrm{MeV}$ and $2 \mathrm{GeV}$, the two expressions are differing by less than $15 \%$, being identical around $600 \mathrm{MeV}$. In fact, the excitation energy is slightly larger (by 10-20\%) for deuteron-induced reactions as for proton-induced reactions, at the same total incident energy (compare Figs. 3 and 19). The number of evaporated neutrons will thus be similar in both cases. These neutrons being more abundant than the INC neutrons, the same holds for the total neutron multiplicity. The approximately equal effects produced by a proton of a given energy and a deuteron of the same energy may, perhaps, be understood on the basis of the following arguments. If there was only elastic scattering between nucleons, a proton of, say $1 \mathrm{GeV}$, would transfer much less energy than two nucleons of $500 \mathrm{MeV}$ each: 


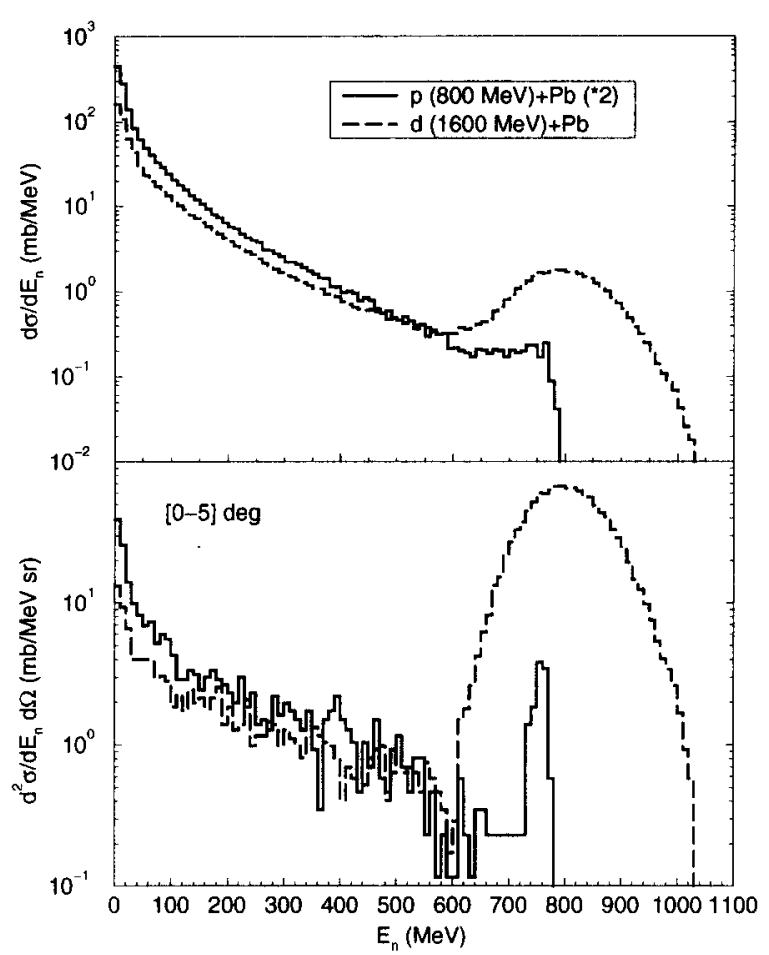

Fig. 20. Neutron double differential cross-sections averaged over angles between $0-5^{\circ}$ (lower part) and integrated over all angles (upper part), in $\mathrm{p}(800 \mathrm{MeV})+\mathrm{Pb}$ reactions (full lines) and $\mathrm{d}(1600 \mathrm{MeV})+\mathrm{Pb}$ reactions (dashed lines). The cross-sections have been multiplied by a factor 2 in the proton-induced case.

the cross-section is lower at $1 \mathrm{GeV}$ and, more importantly, the average scattering angle is much smaller, corresponding to smaller energy loss (the clearing effect mentioned above reduces the difference a little bit). As soon as the energy of the proton is larger than, say $600 \mathrm{MeV}$, the inelastic channel becomes quite important. Delta production is a very efficient process to transfer energy to the target. There seems to be a delicate balance between the two processes in the energy range considered here.

It is also interesting to compare the neutron spectra in proton-induced reactions and in deuteron-induced reactions at the same energy per nucleon $E$. This is illustrated by Fig. 20. For neutron energy $E_{\mathrm{n}}$ less than $\sim 500 \mathrm{MeV}$, the neutrons are less abundant in the second reactions than twice in the first one. At large $E_{\mathrm{n}}$, the neutron yield is conspicuously larger in deuteron-induced reactions, because of the important stripping process. This is even more striking when one focuses on forward angles (see lower part of Fig. 20). Of course, our model neglects the coherent dissociation of the incident deuteron. The latter process is basically due to Coulomb forces and is therefore expected to enhance the neutron yield at high neutron energy and very forward angles. Comparison of our predictions with the recent measurements at SATURNE [27] is in progress and aims at determining the importance of coherent dissociation. 


\section{Summary}

We have presented a general survey of the results of the INC+evaporation model for nucleon and deuteron-induced reactions in the $200-2000 \mathrm{MeV}$ range. Our principal motivation was to provide the physicists who are using spallation reactions for any purpose, with a simple account of the main features of these reactions. That is why we construct pocket formulae for neutron multiplicities and excitation energy. We also show the characteristic values for charged particle multiplicities. Concerning more physical issues than the mere observable multiplicities, we analyzed the decomposition between INC and evaporation contributions. We paid also some attention to the impact parameter dependence of several quantities. We analyzed the evolution of the target parameters in the INC and evaporation steps. We also showed how the neutron double differential cross-sections can be decomposed into evaporation, multiple collision and single collision (elastic and inelastic) components. Since these cross-sections are to be used extensively, a general parametrization with explicit dependence of the various components on target mass and incident energy will presumably be feasible with the extensive measurements presently done at SATURNE [27]. Finally, we studied the deuteron-induced reactions and found that the cascades initiated by the two nucleons inside the deuteron are not additive.

We used the Liège INC model and the evaporation model of Dresner [13]. Many of the results worked out in this paper should not be changed dramatically if other reasonable INC or evaporation models are used. For instance, neutron multiplicities should not change within more than the accuracy of our pocket formulae (of the order of ten percent). Some other quantities may be changed more significantly. In Ref. [5], it is shown that the residue mass distribution and the neutron angular distribution are noticeably different when the Liège or the Bertini INC model is used.

\section{Acknowledgements}

Most of this work was carried out during a stay of one of us (J.C.) at the Service de Physique Nucléaire, DAPNIA, Saclay, for which he is very thankful. The same author wishes also to thank the Division de Physique Théorique at Orsay, where part of this work has been done. We are grateful to the Laboratoire National Saturne for having allowed one of the authors (S.V.) to use the computer facilities and to Dr. S. Leray for her encouragement.

\section{References}

[1] B.B. Cunningham et al., Phys. Rev. 72 (1947) 739.

12| R. Serber, Phys. Rev. 72 (1947) 1114.

13| H.W. Bertini et al., Phys. Rev. 131 (1963) 1801.

|4| F.M. Waterman et al., Phys. Rev. C 8 (1973) 2419.

[5] J. Cugnon, C. Volant and S. Vuillier, Nucl. Phys. A 620 (1997) 475. 
|6] J. Cugnon, Nucl. Phys. A 462 (1987) 751.

[7] G.S. Bauer, 2nd Int. Conf. on Accelerator-Driven Transmutation Technologies and Applications, Kalmar, Sweden, June 3-7 (1996).

[8] C.D. Bowman et al., Nucl. Instr. and Meth. A 320 (1992) 336.

19] S. Andriamonje et al., Phys. Lett. B 348 (1995) 697.

[10] L. Pienkowski et al., Phys. Lett. B 336 (1994) 147.

[11] D. Hilscher, F. Goldenbaum, U. Jahnke, L. Pienkowski, J. Galin, B. Lott and B.Quednau, contribution to the Int. Workshop on Nuclear Methods for Transmutation of Nuclear Waste: Problems, Perspectives, Cooperative Research, Dubna, May 1996, HMI preprint (1996).

[12| V. Barashenkov and V. Toneev, High Energy Interactions of Particles and Atomic Nuclei with Nuclei (Atomizdat, Moscow, 1972).

[13] L. Dresner, Oak Ridge report ORNL-TM-196 (1962).

| 14 | J. Cugnon and M.C. Lemaire, Nucl. Phys. A 489 (1988) 781.

[15] L. Pienkowski et al., Acta Physica Polonica B 25 (1994) 737.

| $16 \mid$ V.F. Weisskopf and D.H. Ewing, Phys. Rev. 57 (1940) 472, 935.

| $17 \mid$ F. Atchison, Proc. Meeting on Targets for Neutron Spallation Sources, Jülich Conf. 34 (1980) 17.

[18| F. Atchison, in Intermediate Energy Nuclear Data: Models and Codes, Proc. of a Specialists' Meeting, Issy-les-Moulineaux (France), OECD, Paris, 1994, p. 201

[19] N. Bohr and J.A. Wheeler, Phys. Rev. 56 (1939) 426.

[20] D. Hilscher and H. Rossner, Ann. Phys. (Fr) (1992) 471.

[21] S. Pearlstein, Nucl. Sci. Eng. 95 (1987) 116.

[22] D.R.F. Cochran, Phys. Rev. D 6 ( 1972) 3085.

[23] X. Blanchard et al., 2nd Int. Conf. on Accelerator-Driven Transmutation Technologies and Applications, Kalmar, Sweden, June 3-7 ( 1996).

[24] S.B. Kaufman and E.P. Steinberg, Phys. Rev. 22 (1980) 167.

[25] S. Cierjacks et al., Phys. Rev. C 36 (1987) 1976.

[26] W.B. Amian et al., Nucl. Sci. Eng. 115 (1993) I.

[27] E. Martinez et al., to be published. 